\title{
ASYMPTOTIC BEHAVIOR OF SOLUTIONS OF HYPERBOLIC INEQUALITIES ${ }^{1}$
}

\author{
BY M. H. PROTTER
}

Communicated by Lipman Bers, April 27, 1962

We consider the asymptotic behavior of solutions of inequalities of the form

$$
|L u|^{2} \leqq c_{1}|u|^{2}+c_{2} \sum_{i=1}^{n}\left|\frac{\partial u}{\partial x_{i}}\right|^{2}+c_{3}\left|\frac{\partial u}{\partial t}\right|^{2}
$$

where

$$
L=A-\frac{\partial^{2}}{\partial t^{2}}+b
$$

and $A$ is a second order elliptic operator. The asymptotic behavior of solutions of parabolic inequalities and related problems have been considered by Agmon and Nirenberg [1], Cohen and Lees [2], Lax [3], and the author [4].

Let $D$ be a bounded domain in $E^{n}$ and suppose $u\left(x_{1}, \cdots, x_{n}, t\right)$ $=u(x, t)$ is a solution of (1.1) in the cylindrical region $R=D \times I$ where $I$ is the half-infinite interval $0 \leqq t<\infty$. We shall study the behavior as $t \rightarrow \infty$ in $R$ of those solutions $u$ which satisfy the additional condition

$$
u=0 \text { on } \Gamma \times I
$$

where $\Gamma$ is the boundary of $D$.

We introduce the notation

$$
\begin{aligned}
(u, v) & =\int_{R} u(x, t) v(x, t) d x d t \\
\|u\| & =(u, u)^{1 / 2} \\
\|u\|_{1}^{2} & =\int_{R} \sum_{i=1}^{n}\left(\frac{\partial u}{\partial x_{i}}\right)^{2} d x d t \\
\|u\|_{D, 1}^{2} & =\int_{D} \sum_{i=1}^{n}\left(\frac{\partial u}{\partial x_{i}}\right)^{2} d x
\end{aligned}
$$

1 This investigation was supported by the United States Air Force through the Air Force Office of Scientific Research of the Air Research and Development Command under Contract No. AF 49(638)-253. 
The elliptic operator $A$ has the form

$$
A=\sum_{i, j=1}^{n} \frac{\partial}{\partial x_{i}}\left(a_{i j} \frac{\partial}{\partial x_{j}}\right), \quad a_{i j}=a_{j i},
$$

where the $a_{i j}=a_{i j}(x, t)$ are $C^{1}$ functions of $x$ and $t$.

A function $v(x, t)$ defined in $R$ is said to satisfy Conditions B if

$$
\begin{array}{rll}
v=0 & \text { on } & \Gamma \times I, \\
\lim _{t \rightarrow \infty} t^{\alpha}\|v\|_{D, 1}=0 & \text { for every } \alpha>0 .
\end{array}
$$

The operator $L$ is said to satisfy Conditions $\mathrm{C}$ if

$$
\begin{aligned}
\frac{\partial}{\partial t}\left(a_{i j}\right) & =O\left(\frac{1}{t}\right) \quad \text { for } i, j=1,2, \cdots, n . \\
\frac{\partial b}{\partial t} & \leqq 0 \quad \text { for all sufficiently large } t .
\end{aligned}
$$

If (1.5) holds and (1.6a) is replaced by

$$
\frac{\partial b}{\partial t}=O\left(t^{-3}\right) .
$$

We say that Conditions $\mathrm{C}^{\prime}$ are satisfied.

Lemma 1. If $v(x, t)$ satisfies Conditions $\mathrm{B}$ and the operator $L$ satisfies Conditions $\mathrm{C}$ or $\mathrm{C}^{\prime}$ then for all sufficiently large $\alpha$ we have

$$
\alpha^{4}\left\|t^{\alpha-2} v\right\|^{2}+\alpha^{2}\left\|t^{\alpha-1} v\right\|_{1}^{2} \leqq m_{0}\left\|t^{\alpha} L v\right\|^{2}
$$

where $m_{0}$ is a positive constant depending only on $L$.

Lemma 2. Under the hypotheses of Lemma 1 we have

$$
\alpha^{1 / 2}\left\|t^{\alpha-1} v_{t}\right\| \leqq m_{1}\left\|t^{\alpha} L v\right\|
$$

for all sufficiently large $\alpha ; m_{1}$ is a positive constant depending only on $L$.

Theorem 1. Let $u(x, t)$ satisfy in $R$ the differential inequality (1.1) and suppose Conditions $\mathrm{B}$ and Conditions $\mathrm{C}$ or $\mathrm{C}^{\prime}$ hold. If in addition

$$
c_{1}(t)=O\left(t^{-2}\right), \quad c_{2}(t), c_{3}(t)=O\left(t^{-1}\right)
$$

then $u \equiv 0$ in $R$.

Theorem 1 follows from Lemmas 1 and 2 by standard arguments.

If we assume that the solution of (1.1) decays more rapidly than stated in Conditions B then the hypotheses on the coefficients of $L$ 
and on $c_{i}(t), i=1,2,3$ may be relaxed considerably.

A function $v(x, t)$ defined in $R$ is said to satisfy Conditions $\mathrm{E}$ if

$$
\begin{array}{rll}
v=0 & \text { on } & \Gamma \times I, \\
\lim _{t \rightarrow \infty} e^{\lambda t}\|v\|_{D, 1}=0 & \text { for every } \lambda>0 .
\end{array}
$$

LemMA 3. Suppose v satisfies Conditions $\mathrm{E}$ and vanishes for $0 \leqq t \leqq \epsilon$ for some $\epsilon>0$. If the coefficients of $L$ have bounded first derivatives then for all sufficiently large $\lambda>0$ we have

$$
\lambda^{4}\left\|e^{\lambda t} v\right\|^{2}+\lambda^{2}\left\|e^{\lambda t} v\right\|_{1}^{2} \leqq m_{2}\left\|e^{\lambda t} L v\right\|^{2}
$$

where $m_{2}$ is a positive constant depending only on $L$.

LEMMA 4. Under the hypotheses of Lemma 3 we have

$$
\lambda^{1 / 2}\left\|e^{\lambda t} v_{t}\right\| \leqq m_{3}\left\|e^{\lambda t} L v\right\|
$$

where $m_{3}$ is a positive constant depending only on $L$.

THEOREM 2. Let $u(x, t)$ satisfy in $R$ the differential inequality (1.1) and suppose Conditions $E$ hold. If the coefficients of $L$ have bounded first derivatives and if $c_{i}(t), i=1,2,3$, are bounded then $u \equiv 0$ in $R$.

\section{BIBLIOGRAPHY}

1. S. Agmon and L. Nirenberg, Properties of solutions of ordinary differential equations in Banach space, New York University Report IMM-NYU 287 (1961).

2. P. J. Cohen and M. Lees, Asymptotic decay of solutions of differential inequalities, Pacific J. Math. 11 (1961), 1235-1249.

3. P. D. Lax, A stability theorem for solutions of abstract differential equations, Comm. Pure Appl. Math. 9 (1956), 747-766.

4. M. H. Protter, Properties of solutions of parabolic equations and inequalities, Canad. J. Math. 13 (1961), 331-345.

University of California, Berkeley 